\title{
Optically Detected Microwave Resonance and Carrier Dynamics in InAs/GaAs Quantum Dots
}

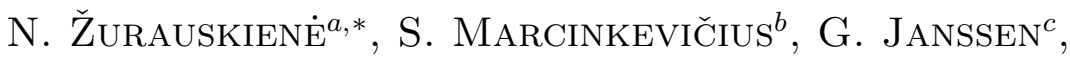 \\ E. Goovaerts ${ }^{c}$, R. NÖtzel ${ }^{d}$, P.M. KoenraAD ${ }^{d}$ \\ AND J.H. WOLTER ${ }^{d}$ \\ ${ }^{a}$ Semiconductor Physics Institute, A. Goštauto 11, Vilnius, Lithuania \\ ${ }^{b}$ Department of Microelectronics and Information Technology \\ Royal Institute of Technology, Electrum 229, 16440 Kista, Sweden \\ ${ }^{c}$ Department of Physics, University of Antwerp \\ Campus Drie Eiken Universiteitsplein 1, 2610 Antwerpen, Belgium \\ ${ }^{d}$ Department of Physics, Eindhoven University of Technology \\ 5600 MB Eindhoven, The Netherlands
}

The properties of small-sized high density InAs/GaAs quantum dots (emitting at $1.25 \mathrm{eV}$ ) are studied by means of optically detected microwave resonance spectroscopy and time resolved photoluminescence techniques. The results are discussed in terms of trapping and thermal escape of the carriers as well as their relaxation and recombination in quantum dots. The data are compared with those recently obtained on shallowly formed InAs quantum dot structures.

PACS numbers: 78.67.Hc, 78.47.+p, 76.70.Hb

\section{Introduction}

Self-assembled quantum dots (QDs) have been intensively studied for the development of infrared photodetectors [1]. QDs provide the ability to absorb normally incident infrared photoexcitation, and long relaxation times ensuring higher responsivity of the detectors. Due to discrete energy levels QDs could be used to detect specific infrared signatures of various objects in space. By engineering of the size and shape of QDs it is possible to achieve wavelength tuning and multicolour detection [2]. To have a detector with high absorption efficiency, it is necessary to have a high density of QDs. Strong and dominant normal inci-

*corresponding author; e-mail: zurausk@pfi.lt 
dent response requires the growth of dots small enough that in-plane confinement leads to only one or two bound electron states [1]. In this paper, properties of high density InAs/GaAs quantum dots with high photoluminescence (PL) peak energy $(1.25 \mathrm{eV})$ indicating small QD dimensions are studied by optically detected microwave resonance (ODMR) and time resolved (TR) photoluminescence techniques. The obtained results are compared with the results obtained on shallowly formed InAs/GaAs QD structures showing close PL peak energy $(1.31 \mathrm{eV})[3,4]$.

\section{Sample specifications and experimental setup}

The investigated quantum dots (sample A, see Ref. [5]) were grown by molecular beam epitaxy on a semi-insulating (100)-GaAs substrate. A $200 \mathrm{~nm}$ GaAs buffer layer was grown at $580^{\circ} \mathrm{C}$, followed by 2.1 monolayers of InAs deposited at $480^{\circ} \mathrm{C}$ at a growth rate of $0.13 \mathrm{ML} / \mathrm{s}$. The QDs were capped with $20 \mathrm{~nm}$ GaAs layer at $480^{\circ} \mathrm{C}$ and $100 \mathrm{~nm}$ of GaAs at $580^{\circ} \mathrm{C}$. The average density of the dots obtained from AFM measurements was $5 \times 10^{11} \mathrm{~cm}^{-2}$. The reference sample B of InAs QDs was grown by $\mathrm{MBE}$ on a $\mathrm{GaAs}$ : $\mathrm{Cr}$ substrate $\left(\right.$ at $490^{\circ} \mathrm{C}$ at a growth rate of $0.285 \mathrm{ML} / \mathrm{s}$ ) [4, 6]. It should be noted that due to relatively high growth temperature of the GaAs capping layer in this sample, the InAs material is partially diffused into the capping layer and therefore a wetting layer (WL) is formed with shallowly formed QDs instead of well-defined dots.

The ODMR spectroscopy was performed using a $95 \mathrm{GHz}$ spectrometer with a $6 \mathrm{~T}$ split-coil superconducting magnet. The microwave-induced changes in the total PL intensity were synchronously detected with the $3 \mathrm{kHz}$ on/off modulation of the applied microwaves at $1.9 \mathrm{~K}$ (for a detailed description of the setup see Ref. [4]).

Carrier dynamics have been studied at 80-240 K temperatures by TRPL technique after excitation with a Ti:sapphire laser (central wavelength $800 \mathrm{~nm}$, pulse duration $130 \mathrm{fs}$, repetition frequency $76 \mathrm{MHz}$ ). A streak camera with an infrared enhanced photocathode combined with a $0.25 \mathrm{~m}$ spectrometer was used for detection. The temporal and spectral resolutions of the measurements were $3 \mathrm{ps}$ and $3 \mathrm{meV}$, respectively. The average excitation intensity was varied from $0.1 \mathrm{~mW}$ up to $30 \mathrm{~mW}$ at a laser spot diameter of $100 \mu \mathrm{m}$.

\section{Results and discussion}

InAs/GaAs QDs were investigated by optically detected microwave resonance spectroscopy. Typical ODMR spectrum of the shallowly formed QDs (sample B) is shown in Fig. 1a. A strong negative signal at low magnetic field was ascribed to cyclotron resonance of the electron in the two-dimensional WL, corresponding to an effective mass of $0.053 m_{0}$. A microwave-induced positive signal at high field was tentatively attributed to magnetic resonance transitions between spin states of the holes confined in shallow dots [4]. No ODMR signal was observed from the well-defined QDs in sample A. The PL spectra for both types of QDs 

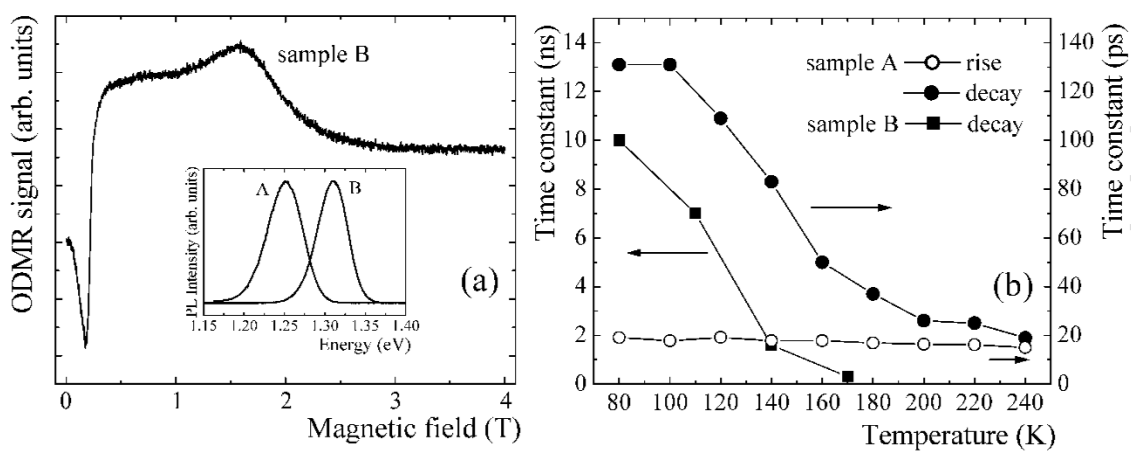

Fig. 1. (a) Microwave spectrum of InAs/GaAs QDs measured when static magnetic field was applied parallel to the growth axis of the sample. Inset presents PL spectra of samples A $(4.2 \mathrm{~K})$ and B $(7.6 \mathrm{~K})$ under cw excitation. (b) Temperature dependence of the PL rise and decay time constants.

are presented in inset of Fig. 1a. High PL peak energies $(1.25 \mathrm{eV}$ for sample A, $1.31 \mathrm{eV}$ for sample B) are the indication of small dimensions of QDs. The electron and hole in QDs of sample B are separated and localized in different regions of the dots $[4,6]$ : the hole is more confined inside the dot, while the electron is weakly bound to it and more probably is found in the WL. This partly explains the relatively long radiative lifetimes, up to $10 \mathrm{~ns}$ at $80 \mathrm{~K}$ (see Fig. 1b and [3]) observed in these structures. Due to the exceptionally long exciton lifetimes, it was possible to perform high microwave frequency $(95 \mathrm{GHz})$ ODMR measurements. In the case of sample A, a shorter PL decay time of $130 \mathrm{ps}$ is found at $80 \mathrm{~K}$ (Fig. 1b). This observation points to a strong confinement of electron and hole in the small QDs, unlike the case of sample B. This is consistent with the lower PL peak energy, and the formation of well-defined QDs, as observed by cross-section scanning tunnelling microscopy (STM) in a sample grown in similar conditions. In this case of a short exciton lifetime, the possibility of ODMR experiments is hampered. To study the carrier dynamics in QDs of sample A, the TRPL measurements were carried out.

Time-integrated PL spectrum measured at several time intervals after the excitation showed shift to lower energies with time. This red shift is attributed to thermal escape of carriers from small QDs (with high ground state emission energies) and subsequent recapture by larger ones using the WL as a transit channel. The PL peak red shift over time decreases with temperature and becomes almost undetectable at $200 \mathrm{~K}$. With increased excitation intensity at $80 \mathrm{~K}$, the PL peak broadens and shifts to higher energies. However, it was impossible to distinguish experimentally peaks of excited states, though theoretical calculations [7] have predicted their existence for such QD dimensions. Most probably, transitions from higher states of larger dots and ground states of smaller ones or from WL states merge to form a single broad PL peak. 
More detailed information on carrier dynamics was obtained by measuring PL transients at particular energies. Typical PL transients at different parts of the emission spectrum are shown in Fig. 2a. After a sharp rise, the PL shows a mono-exponential decay. At low temperatures and low excitation intensities, the PL decay in these well-defined QDs is mainly due to radiative recombination. The comparable emission efficiency of samples A and B indicate that the faster decay in A is not dominated by nonradiative decay, and in this case it could be attributed to strong confinement in small well-defined QDs. According to calculations [7], a significant increase in overlap of electron and hole wave functions was found with decreasing dot size (the limit when the first electron level disappears in WL, as in the case of sample B, was not taken into consideration). However, influence of nonradiative recombination on short decay times in our results also should not be excluded. The rise times, measured at low excitation density, indicate carrier transport in the barriers, and capture and relaxation in the QDs. Figure 1b presents summarized results of characteristic time constants obtained by fitting PL transients with the following expression: $I(t)=I_{0}+A\left\{\exp \left[\left(\tau_{0}-t\right) / \tau_{\text {rise }}\right)\right]-$ $\left.\left.\exp \left[\left(\tau_{0}-t\right) / \tau_{\text {decay }}\right)\right]\right\}$, where $\tau_{\text {rise }}$ and $\tau_{\text {decay }}$ are rise and decay times, respectively, $I_{0}, A$, and $\tau_{0}$ are the fitting constants. The rise time is short (15-20 ps) and roughly independent of temperature. At lower energies within the PL band (larger dots) the rise times are longer (see Fig. 2a). This is probably due to the prolonged carrier transfer into these dots where carriers are first captured by smaller dots, then thermally emitted and recaptured by larger ones. The decay time is also slower in the low energy portion of the spectrum. A rapid decrease in the PL decay time at higher temperatures is attributed to carrier thermal emission and subsequent nonradiative recombination in the barriers and/or WL. A tendency to saturation of decay time at $200 \mathrm{~K}$ and higher temperatures may be due to effective recapture of carriers by the WL or impurities in the QDs. At higher excitation intensities bi-exponential and even more complicated decay is observed due to
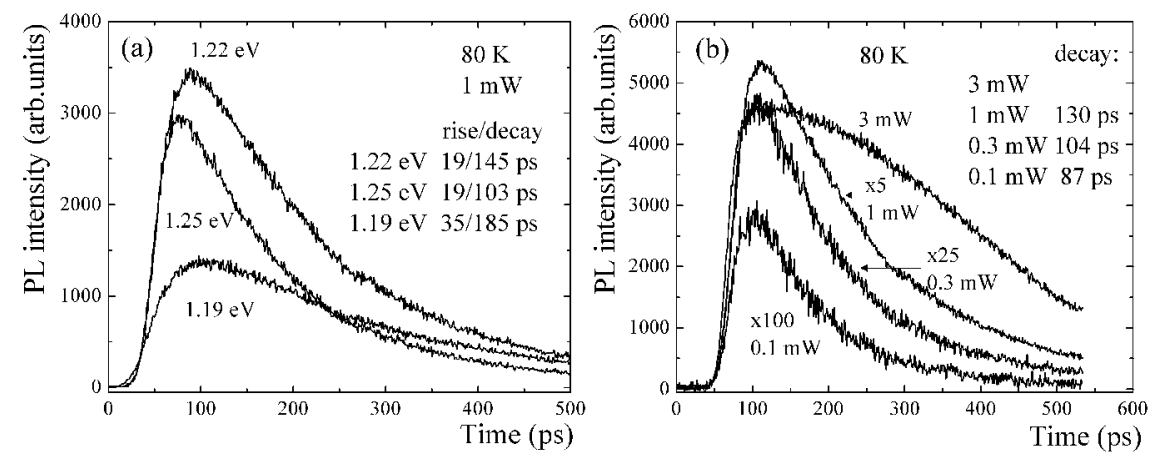

Fig. 2. PL transients of sample A: (a) at different parts of the emission spectrum (at low, high energy side, and at the peak); (b) for different excitation intensities. 
filling of QDs with photoexcited carriers. This is shown in the $3 \mathrm{~mW}$ average excitation curve in Fig. 2b.

In conclusion, we investigated high density InAs/GaAs quantum dot samples both showing a PL at relatively high energy for InAs QDs, close to the wetting layer transition, and obtained significant differences in exciton lifetimes of QDs: $100 \mathrm{ps}$ at $80 \mathrm{~K}$ for sample $\mathrm{A}$, compared to $\approx 10 \mathrm{~ns}$ for sample $\mathrm{B}$. The short PL decay of sample A could result from an increased overlap of hole and electron wave functions in small well-defined QDs, while the long PL decay (sample B) most probably is due to delocalization of the electron wave functions in the WL, thus decreasing this overlap. These properties of small-sized InAs/GaAs QDs should be taken into account in the development of quantum dot-based devices.

\section{Acknowledgments}

Financial support from the Swedish Foundation for International Cooperation in Research and Higher Education (STINT) and the Royal Swedish Academy of Sciences is gratefully acknowledged. We acknowledge also the National Fund for Scientific Research (FWO-Flanders).

\section{References}

[1] H.C. Liu, Opto-Electron. Rev. 11, 1 (2003).

[2] S.Y. Wang, S.C. Chen, S.D. Lin, C.J. Lin, C.P. Lee, Infrared Phys. Technol. 44, $527(2003)$

[3] N. Žurauskienè, S. Marcinkevičius, G. Janssen, E. Goovaerts, A. Bouwen, P.M. Koenraad, J.H. Wolter, Mater. Sci. Forum 384-385, 31 (2002).

[4] G. Janssen, N. Žurauskienè, B. Partoens, B. Van Daele, E. Goovaerts, A. Bouwen, P.M. Koenraad, J.H. Wolter, Phys. Rev. B 68, 045329 (2003).

[5] T. Mano, R. Nötzel, G.J. Hamhuis, T.J. Eijkemans, J.H. Wolter, Appl. Phys. Lett. 81, 1705 (2002).

[6] A.W.E. Minnaert, A.Yu. Silov, W. van der Vleuten, J.E.M. Haverkort, J.H. Wolter, Phys. Rev. B 63, 075303 (2001).

[7] O. Stier, M. Grundmann, D. Bimberg, Phys. Rev. B 59, 5688 (1999). 